\title{
Clinical utility gene card: for incontinentia pigmenti
}

\author{
Francesca Fusco ${ }^{1}$ Alessandra Pescatore ${ }^{1} \cdot$ Julie Steffann $^{2} \cdot$ Jean-Paul Bonnefont $^{2} \cdot$ Judite De Oliveira $^{2}$. \\ Maria Brigida Lioi $^{3} \cdot$ Matilde Valeria Ursini $\mathbb{B}^{1}$
}

Received: 5 February 2019 / Revised: 20 June 2019 / Accepted: 25 June 2019 / Published online: 9 July 2019

(c) European Society of Human Genetics 2019

\section{Disease characteristics}

\subsection{Name of the disease (synonyms)}

Incontinentia Pigmenti; Familial Male-Lethal Type, BlochSulzberger Syndrome.

\subsection{OMIM\# of the disease}

\#308300

\subsection{Name of the analyzed genes or DNA/ chromosome segments}

IKBKG/NEMO (Inhibitor of Kappa light polypeptide gene enhancer in B-cells, Kinase Gamma/NF- $\mathrm{KB}$ Essential MOdulator, NM_001099856.4) located in Xq28 chromosomal region. The corresponding protein is named IKKgamma/NEMO.

\subsection{OMIM\# of the gene(s)}

\#300248

Matilde Valeria Ursini

matildevaleria.ursini@igb.cnr.it

1 Institute of Genetics and Biophysics "Adriano Buzzati-Traverso", IGB-CNR, Naples 80131, Italy

2 Université Paris Descartes-Sorbonne Paris Cité, Imagine INSERM UMR1163, Service de Génétique Moléculaire, Hopital Necker-Enfants Malades, AP-HP, Paris, France

3 Department of Science, University of Basilicata, Potenza 85100, Italy

\subsection{Spectrum of variants}

Incontinentia pigmenti (IP) is an X-linked dominant disease, generally lethal in males, caused by variants of the IKBKG/ NEMO gene (NM_001099856.4), which encodes for IKKgamma/NEMO, essential for NF-kB activation [1-3]. Although the classic IP phenotype is almost entirely restricted to females, occasionally males present an IP phenotype, including the typical skin alterations that are hallmarks of the disease. The rare cases of IP males are postzygotic genetic mosaics for the IKBKG/NEMO variant $[4,5]$ or have a 47 , XXY karyotype (Klinefelter syndrome) [6].

IP variants of the IKBKG/NEMO gene can cause a partial or complete Loss of Function (LoF) of the IKK gamma/ NEMO protein, impairing the NF-kB activation. On the basis of their effect, they are considered hypomorphic variants, when they reduce but do not abolish NF- $\mathrm{kB}$ activation, or amorphic variants, when the NF- $\mathrm{KB}$ activation is completely abolished. While amorphic variants are lethal in males (with the exception of mosaic males), hypomorphic variants have been found in surviving male patients. These males suffer from Anhidrotic Ectodermal Dysplasia, with ImmunoDeficiency (EDA-ID, OMIM\#300291). They are constitutively hemizygous for the IKBKG/NEMO variant, which can be inherited from their IP mother [7].

Around 72 point variants or small indels in the IKBKG/ $N E M O$ gene have been reported so far as the cause of the IP phenotype: 70 identified in IP females [8-10], one in an IP mosaic male [11] and one in an IP female and in her father, IP mosaic for the variant [5].

These variants include small indels $(51 \%)$, and singlenucleotide substitutions (49\%). Moreover, the effects predicted on the mutated protein show that $51 \%$ cause a frameshift, $27 \%$ a premature stop codon, $14 \%$ are missense variants, $7 \%$ are splice-site variants, and only $1 \%$ are inframe amino acid deletions. It is interesting to note that, among the frameshift variants, an intronic point variation NG_009896.1:c.518+866C $>\mathrm{T}$ has been reported as a variant causing a very mild form of IP. This variant creates 
a new splicing donor site, giving rise to a 44-nucleotide pseudo-exon and generating a frameshift and a premature stop in the mutated IKK gamma/NEMO protein [12].

The most frequent pathological variant in IP is an intragenic deletion (IKBKGdel /NEMOdel, found in $78 \%$ of IP female cases) that removes the gene from exon 4 to exon 10 [7]. In addition, other large deletions of all or part of the gene have been reported [13, 14]. Moreover, a postzygotic mosaicism for IKBKGdel /NEMOdel has been reported in IP males [4, 5, 13].

Most IP disease variants have been collected in the public IKBKG/NEMO variants database cataloged in the Leiden Open Variation Database (https://databases.lovd.nl/ shared/genes/IKBKG).

\subsection{Analytical methods}

In a suspected case of IP, different analytical approaches for molecular diagnosis are required to identify the IKBKG/ $N E M O$ alteration. Indeed, if the index case is an IP female the variant is constitutive in the heterozygous state and can be searched for in every cell of the body. Instead, if the index case is an IP male the variant appears postzygotically, leading to embryonic mosaicism, a condition in which two genetically distinct cell populations coexist in the same individual. The timing of the occurrence of the variant plays a key role in the clinical phenotype by determining not only the level of the mosaicism but also the type of affected tissue. In general, cells expressing the IKBKG/NEMO variant allele are selectively eliminated during the life span and finally cleared, making the identification of the IP driven variant in males extremely difficult.

\subsubsection{Looking for constitutive heterozygous IKBKG/NEMO variants in IP female patients}

One strategy for $I K B K G / N E M O$ variant screening is that currently applied on genomic DNA extracted from peripheral blood. The molecular analysis requires specific PCR approaches as reported in a clinical utility card for IP which was published previously (Sanger sequencing with long range and quantitative PCR, LRPCR, and QPCR, respectively) [15]. With the advent of next-generation sequencing (NGS) technology, clinical exome panels are now being increasingly offered by diagnostic laboratories, allowing for a large number of genes to be screened more quickly and more cost effectively. Unfortunately, the complex genomic architecture of the $\mathrm{Xq} 28$ region containing the IKBKG/ NEMO locus, rich in repeated sequences [13, 14] and characterized by two $35 \mathrm{~kb}$ low-copy-repeats sharing $98 \%$ of identity (one containing the IKBKG/NEMO gene and one containing the non-functional IKBKGP/NEMOP pseudogene) makes NGS technology unusable for IP molecular diagnosis. Indeed, as observed by us and recently reported by [12], NGS data analysis pipelines are unable to assign the sequence/copy variant to one of the two copies and a standard capture applied to the IKBKG/NEMO locus results in a decrease in read depth, a decrease in mapping quality and a poor alignment of the reads generated by the pseudogene sequencing which might align with the active gene, resulting in false-positive results.

Despite the advances in sequencing technology, Sanger sequencing remains the gold standard method to analyze the IKBKG/NEMO gene and an adequate NGS strategy needs to be developed in the future to avoid misdiagnosis.

\subsubsection{Looking for mosaic IKBKG/NEMO variants in IP male patients}

The detection of mosaic variants is still a great technical challenge: the low-level mosaicism present in a vulnerable tissue could escape molecular investigation if the methodology employed in relation to IP females is used. Mosaic NEMO/ $I K B K G$ rearrangements and mosaic small variants require specific experimental set-ups to be applied in these cases.

First, in IP males the mosaicism is limited to a small number of mutant cells, which may escape variant detection by the standard technologies on account of the level of resolution. Detection of mosaicism in human disease is indeed challenging because mosaicism may be tissue-specific or tissue-limited. In IP males the choice of tissue is suggested by the recognition of a suspected phenotype (e.g., a biopsy from skin with IP lesions) and from an analysis of multiple tissues performed to rule out low-level mosaicism: blood, fresh skin, saliva, and sperm samples can be analyzed with the methodology used in relation to IP females (Sanger sequencing with LRPCR and QPCR). As reported in ref. [5], for molecular diagnosis in IP males, the blood is not an appropriate tissue to be investigated. A skin biopsy along the lesions in boys and sperm in the adult males are the appropriate biological materials for this molecular diagnosis. IKBKG/NEMO variants can be identified in the blood only in samples taken from IP male newborns.

\subsection{Analytical validation}

Analyses of known positive and negative control samples are required for the validation of any diagnostic genetic test procedure. Sequence alterations that are variants that affect function are bidirectionally sequenced on the IKBKG/NEMO gene specific template. Moreover, these variants can be further investigated by in silico analysis; confirmation of the segregation of the variant in the parents is recommended. Furthermore, the identified variants should be checked against existing entries in the SNP databases. 
Mosaicism identification is usually a multistep process, extensive, expensive and time consuming. Usually more than one technique is used to recognize mosaicism and additional methods are needed to confirm the finding. The degree of mosaicism of any IKBKG/NEMO variant can be evaluated from different tissue DNA sources by quantifying the copy number variations in the IP locus (when any rearrangement is detected) by QPCR using locus-specific probes [14] or a TaqMan SNP Genotyping assay when a specific point variant is identified. In this case an allelespecific QPCR assay can be used for quantification of the IKBKG/NEMO variant when applicable (e.g., in the case of a single-nucleotide variant) [5]. Overall, these findings suggest the following observations:

1. Peripheral blood is not the appropriate tissue to reveal the somatic mosaicism in IP males, although it represents the main source of DNA in routine IP diagnosis.

2. Genetic investigation in sperm DNA is recommended because the gonadal cells carrying the IKBKG/NEMO variant are able to survive differently from other cells (fibroblasts and blood cells).

The tissues used in this analysis have, consequently, a different role: skin with lesions, sperm and urine are considered positive tissues, where it is more likely to find the variant; blood and unaffected skin are, instead, the tissues to be used as a negative control.

Finally, using clinical and diagnostic data, a genotype-phenotype correlation will be assessed to discuss and/or predict the clinical consequences of mosaicism.

\subsection{Estimated frequency of the disease}

(Incidence at birth ("birth prevalence") or population prevalence. If known to be variable between ethnic groups, please report):

Incontinentia pigmenti (IP; OMIM\#308300) is a rare multisystemic genomic disorder with an estimated birth prevalence in European population of 1.2/100,000 [16].

\subsection{Diagnostic setting}

\begin{tabular}{lll}
\hline & Yes. & No. \\
A. (Differential) diagnosis & $\bigotimes$ & $\square$ \\
B. Predictive testing & $\bigotimes$ & $\square$ \\
C. Risk assessment in relatives & $\bigotimes$ & $\square$ \\
D. Prenatal & $\bigotimes$ & $\square$ \\
\hline
\end{tabular}

\section{Comment:}

A first comment relates to the differential diagnostics.

Some conditions with skin manifestations along Blaschko's lines may be confused with IP [17]. HYPOMELANOSIS OF ITO (HMI), which does not represent a distinct entity but is rather a symptom of many different states of mosaicism, is frequently misdiagnosed as IP. HMI presents with skin signs characterized by unilateral or bilateral macular hypopigmented whorls, streaks, and patches which are described as the "negative pattern" of the hyperpigmented lesions of IP. A definitive HMI diagnosis could be confirmed by routine genetics evaluation.

A second comment concerns the Predictive Test.

IP (OMIM\#308300), presents a wide phenotypic variability ranging from a mild dermatosis, appearing soon after birth, to a severe neurological and/or ocular impairment $[15,18,19]$. The severity of the disease is related to the presence of central nervous system (CNS) manifestations (30\% of cases) ranging from a neonatal single-seizure episode to severe motor and intellectual disability and eye abnormalities (27\% of cases) including retinal vascular abnormalities [15, 20-23]. The variability is extreme; indeed, in some cases, the IP phenotype in relatives of the index case is mild and revealed only through genetic testing. In such cases, the molecular test has a predictive value by indicating the carriers in the IP family. The recent establishment of a biobank for IP (IPGB, http://www.igb. cnr.it/ipgb) has improved the deep phenotyping analysis in all family members and has revealed an extensive intrafamilial heterogeneity with both mild and severe forms in the same family. However, as the mechanisms causing such heterogeneity are still unknown, the genetic result does not have an appropriate predictive value because it does not offer an accurate prediction of the severity of the disease phenotype.

Moreover, data from the IPGB biobank shows that $>75 \%$ of IP females and all IP males are sporadic cases and, consequently, their relatives (excluding offspring) have no elevated risk of developing IP. Nevertheless, the risk of a recurrence of the disease in the siblings of a sporadic index case due to allelic mosaicism in the mother's oocytes or father's sperm cannot be excluded [5].

The identification of the variant that affects function will permit detection in the family and prenatal diagnosis. It is recommended to characterize the variant in the index patient before testing at-risk relatives.

On the basis of the effects of the IKBKG/NEMO variant on the NF- $\mathrm{KB}$ activation, the risk of having a son with EDAID must be evaluated. Indeed, hypomorphic constitutive variants in the IKBKG/NEMO gene are not lethal in the male fetus and cause Anhidrotic Ectodermal Dysplasia with ImmunoDeficiency (EDAID, OMIM\#300291) in any 
hemizygous sons and IP (IP, OMIM\#308300) in any heterozygous daughters.

Interestingly, in the case of a familial inheritance of EDA-ID, the IKBKG/NEMO variants can cause IP in the mother, and EDA-ID in the hemizygous male (her probandchild). It is interesting to note that the IP mother phenotype, when reported, is very mild and can occasionally escape clinical diagnosis [7].

\section{Test characteristics}

$\begin{array}{ll}\begin{array}{l}\text { Genotype or A: true positives } \\ \text { disease }\end{array} & \begin{array}{l}\text { C: false } \\ \text { negatives }\end{array} \\ \text { Present absent B: false positives } & \text { D: true } \\ & \text { negatives }\end{array}$

test

$\begin{array}{ccll}\text { pos. A } & \text { B } & \text { Sensitivity: } & \text { A } /(A+C) \\ & & \text { Specificity: } & \mathrm{D} /(\mathrm{D}+\mathrm{B}) \\ \text { neg. C } & \text { D } & \begin{array}{l}\text { Pos. predict. value: } \\ \text { Neg. predict. value: }\end{array} & \mathrm{D} /(\mathrm{A} / \mathrm{C}+\mathrm{B}) \\ & & \end{array}$

\subsection{Analytical sensitivity}

(proportion of positive tests if the genotype is present)

Close to $100 \%$. By analyzing the data from IPGB the analytic sensitivity of the test for the recurrent deletion (IKBKGdel/NEMOdel) is $79.2 \%$, when combined with Sanger sequencing of the coding regions and splice sites, and reaches 83\%, when QPCR is used to detect other large rearrangements.

Comment: Quantitative PCR (QPCR) does not detect point variants in the gene nor other genomic alterations outside the IP locus. Depending on the technique and methods used in each laboratory, the sensitivity may vary.

It is recommended to scan SNP databases periodically to check for the identification of novel SNPs, prone to interfere with primer hybridization.

Unfortunately, in IP males the detection of mosaicism is underestimated because it is dependent on the variant identification per se. Moreover, DNA from a selected tissue sample still expressing the causative $N E M O / I K B K G$ variant (e.g., a skin biopsy or sperm sample) would be optimal for the testing and always preferable to using blood samples.

New sequencing technologies constitute a promising methodological solution for mosaicism detection in the coming years and revisions of the current diagnostic protocols are necessary to increase the detection rate of unrevealed mosaicism events.

\subsection{Analytical specificity}

(proportion of negative tests if the genotype is not present)

The analytical specificity reaches nearly $100 \%$. Falsepositive results are rare and could be explained by misinterpreting known or unknown variants: some SNVs were historically classified as pathogenic, and should be reclassified as polymorphisms due to their frequency in the general population.

The main concern is the occasional detection of exonic variants of uncertain significance, whose disease-causing effect is often difficult to demonstrate.

\subsection{Clinical sensitivity}

(proportion of positive tests if the disease is present) The clinical sensitivity is dependent on variable factors such as age or family history. In such cases a general statement should be given, even if a quantification can only be made case by case.

If a correct clinical diagnosis has been made the disease-causing IKBKG/NEMO variant is identified in close to $83 \%$ of cases in IP females. However, $~ 17 \%$ of patients referred for molecular diagnosis due to a suspicion of IP are negative for known disease-causing IKBKG/ $N E M O$ variants. We cannot exclude the possibility that they have alterations in IP locus regions that are outside the region investigated by the current tests (regulatory regions, introns, etc).

The clinical sensitivity depends on variable factors such as age or family history. Moreover, IP patients (both IP females and males) have a heterogeneous clinical presentation and, while they always have typical linear skin lesions (starting at birth and spontaneously evolving in four overlapping dermatological stages), they inconsistently exhibit other defects, either ophthalmological (strabismus, cataracts, optic atrophy, retinal vascular pigmentary abnormalities, or microphthalmia), odontological (partial anodontia, delayed dentition, cone/ pegshaped teeth, or impactions), or neurological (seizures, spastic paralysis, motor, and mental retardation ormicrocephaly). The severity of these additional clinical signs is variable $[1,20,21]$.

\subsection{Clinical specificity}

(proportion of negative tests if the disease is not present) The clinical specificity can be dependent on variable factors such as age or family history. In such cases a general statement should be given, even if a quantification can only be made case by case.

The clinical specificity, that is the proportion of negative tests if the disease is not present, is around $100 \%$ for 
relatives of the IP index case in whom the alteration has been identified.

\subsection{Positive clinical predictive value}

(life time risk of developing the disease if the test is positive)

On the basis of studies of large pedigrees, most, if not all, IP patients with a positive test are penetrant for the condition.

A wide variability, ranging from a mild dermatosis to a severe neurological and/or ocular impairment, has been observed [15, 20, 21]. The severity of the disease is related to the presence of CNS manifestations (30\% of cases) or of eye abnormalities ( $27 \%$ of cases) including retinal vascular abnormalities [15, 21-23].

In IP females and males skin lesions are almost always found, tooth and eye anomalies are detected in more than $50 \%$ of cases, and a CNS involvement is present in 10-30\% of cases [15, 20, 21].

Both female and male individuals who result positive for NEMO/IKBKG pathogenic variants should receive genetic counseling regarding the testing, inheritance pattern, disease diagnosis and prognosis, and risk of recurrence.

\subsection{Negative clinical predictive value}

(Probability of not developing the disease if the test is negative). The increased risk for a non-affected person of not developing the disease is close to $100 \%$.

\section{Clinical utility}

\section{1 (Differential) diagnostics: Is the tested person clinically affected?}

(To be answered if "A" was marked in 1,9)

\subsubsection{Can a diagnosis be made other than through a genetic test?}

$\begin{array}{lll}\text { No. } & \square \text { (continue with 3.1.4) } \\ \text { Yes. } & \bigotimes \\ & \text { Clinically. } & \square \\ & \text { Imaging. } & \square \\ & \text { Endoscopy. } & \square \\ & \text { Biochemistry. } & \square \\ & \text { Electrophysiology. } & \square \\ & \text { Other (please describe): } & \text { X-skin histology } \\ & \end{array}$

\subsubsection{Describe the burden of alternative diagnostic methods on the patient}

Clinical diagnosis (combining family history and physical examination) associated with genetic testing is required to confirm the diagnosis. Moreover, the identification of the disease-causing variant in the index case also simplifies the predictive test in family members. IP can be diagnosed clinically, but not solely, using criteria for the classification of IP which has established that affected females have a history of perinatal blistering and skin lesions at at least one of the four stages. Histological features of the skin can assist the diagnosis at least in males with IP: spongiosis (stage1) dyskeratosis (stage 2) and free melanin in the dermis (stage 3) reflect keratinocyte apoptosis and can be included as major criteria of IP [20].

In addition to the classic skin findings, the clinical diagnosis may be based on findings relating to the skin and skin appendages (hair and nails) and to an abnormal dentition. Specific eye and CNS involvement are less common but should be evaluated. A clinical assessment including a full pedigree, history and complete physical examination by a clinical geneticist is recommended. Molecular testing can be confirmatory, but is not obligatory in affected females. However, it is essential in cases of males with a clinical suspicion of IP.

\subsubsection{How is the cost effectiveness of alternative diagnostic methods to be judged?}

A physical examination and skin biopsy are cost-effective methods compared to genetic testing. In our experience, making a diagnosis of IP is inevitably a requirement for genetic testing. For males with IP the genetic test is more expensive because it requires specific competences and infrastructures for the skin biopsy and analysis of DNA from different tissues.

3.1.4 Will the disease management be influenced by the result of a genetic test?

No. $\bigotimes$

There is no specific treatment for IP.

The disease management is not affected by the genetic diagnosis if the clinical diagnosis is unambiguous. However, a positive test should result in cascade testing of atrisk female relatives, raising the awareness in those females who might present very mild or ambiguous signs of IP. In no case is a positive-genetic test indicative of the disease outcome, either severe or mild. 
Yes.

Therapy (please describe)

Prognosis (please describe)

Management (please describe)

3.2 Predictive setting: Is the tested person clinically unaffected but carrying an increased risk based on family history

(To be answered if "B" was marked in 1.9)

3.2.1 Will the result of a genetic test influence lifestyle and prevention?

While the genetic test result does not influence lifestyle it is essential for prevention and genetic counseling.

If the test result is positive (please describe)

Patients are typically diagnosed due to phenotypic skin manifestations around birth [1]. In $30 \%$ of cases, a neonatal single-seizure episode and retinal vascular abnormalities can indicate the onset of a severe phenotype [15, 19-21]. Positive predictive testing can also have a significant effect on the family. In addition, early diagnosis can prompt carrier testing in the mother (and subsequent familial cascade screening if the mother is positive for a disease-causing $I K B K G / N E M O$ variant) and gives parents the option of preimplantation or prenatal testing.

For IP males the risk of germline mosaicism needs to be considered.

If the test result is negative (please describe) No

3.2.2 Which options in relation to lifestyle and prevention does a person at-risk have if no genetic testing has been performed (please describe)?

Lifestyle and prevention should be exactly the same as in the above case if the clinical diagnosis is certain.

\subsection{Has genetic risk assessment in the family members of a diseased person been performed?/ls genetic risk assessment in the family members of a diseased person recommended?}

(To be answered if "C" was marked in 1.9)

3.3.1 Does the result of a genetic test resolve the genetic situation in that family?

Yes. Genetic testing is necessary for genetic risk assessment in siblings.
3.3.2 Can genetic testing in the index patient mean that genetic or other tests can be avoided in other family members?

A conclusive genetic test in an index patient (an IP female) would characterize the IKBKG/NEMO-causing variant, which is almost always present, in that family. As such, this may enable maternally related females who are manifesting a similar disease to avoid other invasive tests. The mother of the index patient should be tested. A conclusive genetic test in an index patient (an IP male) would characterize only the female offspring that are at risk.

3.3.3 Does a positive-genetic test mean that the index patient may enable a predictive test in a family member?

Following a positive test result in a female index case, the mother should be tested for that IKBKG/NEMO variant. However, negative peripheral blood DNA testing results do not rule out the possibility of maternal or paternal gonadal mosaicism [5] and should be discussed with a genetic counselor. If the mother is affected, at-risk relatives should be tested to determine carrier/disease status.

\subsection{Prenatal diagnosis}

(To be answered if "D" was marked in 1.9)

3.4.1 Does a positive-genetic test mean that the index patient may enable a prenatal diagnosis?

Yes. Prenatal testing is usually only considered when the index patient's mother is a known or suspected carrier of IP, and the disease-causing IP variant is known. However, germline mosaicism in the mother and father should be considered as a risk factor.

\section{If applicable, what are the further consequences of testing?}

Please assume that the result of a genetic test has no immediate medical consequences. Is there any evidence that a genetic test is nevertheless useful for the patient or his/her relatives? (Please describe)

Although the result of a genetic test has no immediate medical consequences because there is no specific therapy for IP, it will be helpful to investigate the presence of learning disabilities (LD) through periodic assessments of learning skills in order to apply specific targeted therapeutic strategies. Indeed, Pizzamiglio et al. [23] reported on the high prevalence of LD in individuals with IP without intellectual deficiencies and also on the importance of an early assessment to prevent 
any worsening of the defect. Finally, an early genetic diagnosis may influence future reproductive choices and underpin informative genetic counseling.

Acknowledgements We would like to thank the patients, and their families and physicians. We are also grateful to the Italian Incontinentia Pigmenti Association (www.incontinentiapigmenti. it), the French Incontinentia Pigmenti Association (http://incontinentiapigmenti.fr), and the Incontinentia Pigmenti International Foundation (www.ipif. org). This work was supported by the Incontinentia Pigmenti International Foundation (IPIF, http://www.ipif.org), the Italian Incontinentia Pigmenti Association (IPASSI, http://www.incontinentiapigmenti.it), the French Incontinentia Pigmenti Association (FIP, http://incontinentiapigmenti.fr), by CNR-DSB Progetto Bandiera "InterOmics" grant to Fusco and Ursini and by the InBioMed project grant (ARS01_01081_ PON/PNR 2015-20 MIUR to MB Lioi and to MV Ursini). We would like to thank Ahmed EL-Sharkawy for revising the paper.

\section{Compliance with ethical standards}

Conflict of interest The authors declare that they have no conflict of interest.

Publisher's note: Springer Nature remains neutral with regard to jurisdictional claims in published maps and institutional affiliations.

\section{References}

1. Scheuerle AE, Ursini MV. Incontinentia pigmenti. In: Adam MP, Ardinger HH, Pagon RA, Wallace SE, Bean LJH, Stephens K, Amemiya A (eds). GeneReviews ${ }^{\circledR}$ [Internet]. Seattle (WA): University of Washington, Seattle; 1993-2019.

2. Hayden MS, Ghosh S. Signaling to NF-kappaB. Genes Dev. 2004; 18:2195-224.

3. Nelson DL. NEMO, NF kappaB signaling and incontinentia pigmenti. Curr Opin Genet Dev. 2006;16:282-8.

4. Fusco F, Fimiani G, Tadini G, Michele D, Ursini MV. Clinical diagnosis of incontinentia pigmenti in a cohort of male patients. $\mathrm{J}$ Am Acad Dermatol. 2007;56:264-7.

5. Fusco F, Conte MI, Diociaiuti A, Bigoni S, Branda MF, Ferlini A, et al. Unusual father-to-daughter transmission of incontinentia pigmenti due to mosaicism in IP males. Pediatrics. 2017;140: e20162950. https://doi.org/10.1542/peds.2016-2950.

6. Kenwrick S, Woffendin H, Jakins T, Shuttleworth SG, Mayer E, Greenhalgh L, et al. International IP Consortium. Survival of male patients with incontinentia pigmenti carrying a lethal mutation can be explained by somatic mosaicism or Klinefelter syndrome. Am J Hum Genet. 2001;69:1210-7.

7. Fusco F, Pescatore A, Conte MI, Mirabelli P, Paciolla M, Esposito E, et al. EDA-ID and IP, two faces of the same coin: how the same IKBKG/NEMO mutation affecting the NF- $\kappa$ B pathway can cause immunodeficiency and/or inflammation. Int Rev Immunol. 2015;34:44.
8. Fusco F, Pescatore A, Bal E, Ghoul A, Paciolla M, Lioi MB, et al. Alterations of the IKBKG locus and diseases: an update and a report of 13 novel mutations. Hum Mutat. 2008;29:595-604.

9. Conte MI, Pescatore A, Paciolla M, Esposito E, Miano MG, Lioi $\mathrm{MB}$, et al. Insight into IKBKG/NEMO locus: report of new mutations and complex genomic rearrangements leading to incontinentia pigmenti disease. Hum Mutat. 2014;35: 165-77.

10. Bal E, Laplantine E, Hamel Y, Dubosclard V, Boisson B, Pescatore $A$, et al. Lack of interaction between NEMO and SHARPIN impairs linear ubiquitination and NF- $\mathrm{KB}$ activation and leads to incontinentia pigmenti. J Allergy Clin Immunol. 2017;140:1671-82.

11. Hull S, Arno G, Thomson P, Mutch S, Webster AR, Rai H, et al. Somatic mosaicism of a novel IKBKG mutation in a male patient with incontinentia pigmenti. Am J Med Genet A. 2015; 167:1601-4.

12. Boisson B, Honda Y, Ajiro M, Bustamante J, Bendavid M, Gennery AR, et al. Rescue of recurrent deep intronic mutation underlying cell type-dependent quantitative NEMO deficiency. J Clin Invest. 2018;124011. https://doi.org/10.1172/JCI124011.

13. Fusco F, Paciolla M, Pescatore A, Lioi MB, Ayuso C, Faravelli F, et al. Microdeletion/duplication at the Xq28 IP locus causes a de novo IKBKG/NEMO/IKKgamma exon4_10 deletion in families with Incontinentia Pigmenti. Hum Mutat. 2009; 30:1284-91.

14. Fusco F, Paciolla M, Napolitano F, Pescatore A, D'Addario I, Bal E, et al. Genomic architecture at the Incontinentia Pigmenti locus favours de novo pathological alleles through different mechanisms. Hum Mol Genet. 2012;21:1260-71. https://doi.org/10.1093/ hmg/ddr55.

15. Fusco F, Pescatore A, Steffann J, Royer G, Bonnefont JP, Ursini MV Clinical Utility Gene Card for: incontinentia pigmenti. Eur J Hum Genet. 2013;21. https://doi.org/10.1038/ejhg.2012.227.

16. Orphanet Report Series-Prevalence of rare diseases: Bibliographic data-June 2018-Number 1.

17. Donnai D. Incontinentia pigmenti. In: Cassidy S, Allanson JE, (eds.). Management of genetic syndromes. 2nd edn. Hoboken, NJ: Wiley-Liss; 2005. p. 309-14.

18. Landy SJ, Donnai D. Incontinentia pigmenti (Bloch-Sulzberger syndrome). J Med Genet. 1993;30:53-9.

19. Minic S, Trpinac D, Obradovic M. Incontinentia pigmenti diagnostic criteria update. Clin Genet. 2014;85:536-42.

20. Hadj-Rabia S, Froidevaux D, Bodak N, Hamel-Teillac D, Smahi A, Touil Y, et al. Clinical study of 40 cases of incontinentia pigmenti. Arch Dermatol. 2003;139:1163-70.

21. Fusco F, Bardaro T, Fimiani G, Mercadante V, Miano MG, Falco $\mathrm{G}$, et al. Molecular analysis of the genetic defect in a large cohort of IP patients and identification of novel NEMO mutations interfering with NF-kappaB activation. Hum Mol Genet. 2004; 13:1763-73.

22. Pizzamiglio MR, Piccardi L, Bianchini F, Canzano L, Palermo L, Fusco F, et al. Incontinentia pigmenti: learning disabilities are a fundamental hallmark of the disease. PLoS One 2014;9: e87771. https://doi.org/10.1371/journal.pone.0087771. eCollection 2014.

23. Meuwissen ME, Mancini GM. Neurological findings in incontinentia pigmenti: a review. Eur J Med Genet. 2012;55:323-31. 